\title{
Optimal Detection of Sentinel Lymph Node Metastases by Intraoperative Radioactive Threshold and Molecular Analysis in Patients with Melanoma
}

\author{
Gianpiero Manca ${ }^{1}$, Antonella Romanini ${ }^{2}$, Daniela Pellegrino ${ }^{1}$, Elisa Borsò ${ }^{1}$, Marianna Rondini ${ }^{2}$, Cinzia Orlandini², \\ Vanna Zucchi ${ }^{3}$, Francesco Pasqualetti ${ }^{4}$, and Giuliano Mariani ${ }^{1}$ \\ ${ }^{1}$ Regional Center of Nuclear Medicine, University of Pisa Medical School, Pisa, Italy; ${ }^{2}$ Division of Medical Oncology, University \\ Hospital, Pisa, Italy; ${ }^{3}$ Pathology Unit, General Hospital, Livorno, Italy; and ${ }^{4}$ Division of Radiotherapy, University Hospital, Pisa, Italy
}

The aim of this study was to optimize a protocol for radioguided biopsy of the sentinel lymph node (SLN) in patients with melanoma. The protocol was based on a combination of ex vivo counting of the nodes detected intraoperatively and analysis of the harvested nodes by hematoxylin and eosin staining plus immunohistochemistry (conventional histopathology [PATH]) and by molecular biology (reverse-transcriptase polymerase chain reaction [RT-PCR]). Methods: A total of 124 patients with primary clinical stage I-II (according to the American Joint Committee on Cancer) cutaneous melanoma underwent successful radioguided SLN biopsy. SLNs harvested for analysis included any additional nodes whose ex vivo counting rate exceeded $20 \%$ of the hottest node. All removed SLNs were examined by conventional PATH and with RT-PCR analysis for the expression of messenger RNA for tyrosinase and the melanoma antigens recognized by T cells. Complete lymph node dissection (CLND) was performed only in the case of SLN metastasis detected by PATH. Different combinations of the intraoperative parameters (only the hottest node and all nodes harvested) and of analysis (PATH and RTPCR) were tested as predictors of clinical outcome on the basis of long-term follow-up (12-81 mo; median, 55 mo). Results: A total of 197 SLNs were harvested, 41 of which harbored metastasis as detected by RT-PCR analysis; PATH detected metastasis in only 24 of 41 metastatic SLNs. In 5 of 41 instances, metastasis was not in the hottest SLN. The main factor determining correct classification of the SLN status was RT-PCR, which significantly improved detection of metastasis, even if applied only to the hottest node $(P<0.0001$ vs. PATH analysis of either the hottest SLN or all nodes above the $20 \%$ threshold). Metastatic disease recurred locally in 5 patients who had not undergone CLND; RT-PCR analysis showed metastasis in 4 of these patients. The false-negative rate of SLN biopsy progressively decreased when applying PATH only to the hottest node (32.1\%), additional RT-PCR to the hottest node (21.4\%), PATH to all nodes $(17.9 \%)$, and RT-PCR to all nodes $(3.6 \%, P=0.015$ vs. PATH analysis of

Received Jun. 23, 2008; revision accepted Aug. 7, 2008.

For correspondence or reprints contact: Gianpiero Manca, Regional Center of Nuclear Medicine, University of Pisa Medical School, Via Roma, 67 I-56126 Pisa, Italy.

E-mail: g.manca@med.unipi.it

COPYRIGHT @ 2008 by the Society of Nuclear Medicine, Inc. only the hottest SLN). Conclusion: On the basis of long-term follow-up (the gold standard for final clinical outcome of SLN biopsy), both $20 \%$ threshold and RT-PCR analysis should be applied for optimal detection of nodal metastases in patients with melanoma.

Key Words: malignant cutaneous melanoma; radioguided sentinel lymph node biopsy; intraoperative ex-vivo counting; radioactive threshold; molecular analysis; clinical outcome

J Nucl Med 2008; 49:1769-1775

DOI: 10.2967/jnumed.108.055350

$\mathbf{L}$ ymphatic mapping and sentinel lymph node (SLN) biopsy have rapidly emerged as the standard of care for patients with malignant cutaneous melanoma, as the procedure readily identifies those patients who may benefit from immediate complete lymph node dissection (CLND) of the lymphatic basin at risk for metastasis $(1,2)$. In fact, the diseasefree survival rate is significantly greater when CLND is performed immediately after a positive SLN biopsy than when nodal metastasis becomes clinically evident $(78.5 \%$ vs. $73 \%$, $P=0.009$, at a median follow-up of $59.8 \mathrm{mo}$ ) (3).

Definition of the SLN as a biologic rather than an anatomic entity (i.e., not simply the node that lies closer to the tumor, but the regional node to which a tumor would first metastasize (4)) suggests some technical difficulties in the identification of the true SLN in the surgical field. In fact, multiple radioactive lymph nodes in the same lymphatic basin are often detected by lymphoscintigraphy or by intraoperative $\gamma$-probe counting. Especially if the progress of the radiocolloid has not been adequately monitored during lymphoscintigraphy, it is not always clear whether all these radioactive lymph nodes represent true SLNs or whether they simply are secondechelon nodes sequentially visualized by radiocolloid particles passing through the first, true SLN.

Therefore, different approaches for performing radioguided SLN biopsy have been described on the basis of the level of 
radioactivity detected within the nodes, either intraoperatively or by immediate ex vivo counting. Some authors base SLN identification on the absolute number of counts in the nodes, whereas others consider the ratio of the in vivo or ex vivo radioactive counts in the node relative to background or to neighboring non-SLNs $(5,6)$. All such modalities are somewhat arbitrary, and debate is still open regarding the optimal strategy for removing radioactive lymph nodes for pathologic examination. An empiric threshold corresponding to $10 \%$ or more of the counting rate in the hottest SLN is widely reported in the literature (7), but it may lead to the superfluous removal of multiple non-SLNs (4).

The false-negative rate of SLN biopsy (i.e., the proportion of patients in whom metastatic disease appears during follow-up in a lymphatic basin that had been classified as disease-free on the basis of a negative SLN biopsy) ranges from $10 \%$ to $15 \%$ in patients with melanoma (8). Part of these false-negative cases is due to inadequate histopathologic analysis, such as hematoxylin and eosin (H\&E) staining alone (9). Immunohistochemistry (IHC), and especially molecular biology techniques (e.g., reverse-transcriptase polymerase chain reaction [RT-PCR]), improve dramatically the capability of detecting microscopic metastatic disease in SLNs of patients with melanoma. Nevertheless, although IHC with antibodies to the S-100 (10) or HMB-45 antigens (11) has 10\%-30\% higher sensitivity for identifying micrometastases than does H\&E staining (9), it still has a sizable false-negative rate $(6 \%-11.5 \%)(12-14)$. On the other hand, the RT-PCR technique upstages an additional $13 \%-30 \%$ of patients in whom SLNs were negative when analyzed by both $\mathrm{H} \& \mathrm{E}$ and IHC staining (15-18).

In this work, we define a protocol for radioguided SLN biopsy in patients with melanoma - a protocol that minimizes the number of radioactive lymph nodes removed and at the same time minimizes the risk of missing microscopic metastatic disease in the nodes removed. In particular, our goal was to verify that combining removal of radioactive lymph nodes that were $20 \%$ or more of the ex vivo counting rate in the hottest lymph node with molecular analysis in addition to $\mathrm{H} \& \mathrm{E}$ and IHC staining represents the best compromise in this setting. Long-term follow-up represented the gold standard for final evaluation of the clinical outcome of the procedure.

\section{MATERIALS AND METHODS}

\section{Patients}

The study includes 124 consecutive patients with cutaneous melanoma who underwent successful radioguided SLN biopsy. Informed written consent was obtained for the protocol, which was approved by the Ethical Committee of the University Hospital of Pisa. The primary melanomas were either a Breslow thickness of less than $1 \mathrm{~mm}$ and a Clark level III-V (clinical stage I and II, according to the American Joint Committee on Cancer) (19) or a Breslow thickness of $1 \mathrm{~mm}$ or more (stage II), with or without ulceration. Patient characteristics are summarized in Table 1. According to current established guidelines, clinical decisions for further surgical or other medical treatment were made solely on the basis of PATH
TABLE 1

Clinical Characteristics of the 124 Patients Included in Study

\begin{tabular}{lc}
\hline \multicolumn{1}{c}{ Characteristic } & Value \\
\hline Median age (y) & 56 (range, 20-81) \\
Sex (\%) & 53.20 \\
Male & 46.80 \\
Female & \\
Primary tumor site (\%) & 56.60 \\
Nonextremities & 43.40 \\
Extremities & \\
Histology (\%) & 53.60 \\
Superficial spreading & 46.40 \\
Other types & \\
Breslow thickness & $2.0 \pm 1.18$ \\
Mean \pm SD (mm) & (range, $0.4-6.0)$ \\
Median (mm) & 62.70 \\
\hline$<2.0$ mm (\%) & 31.70 \\
$2.01-4.0$ mm (\%) & 5.60 \\
$>4.0$ mm (\%) & \\
Clark stage (\%) & 34.60 \\
I-III & 65.40 \\
IV-V & \\
Ulceration (\%) & 14.20 \\
Present & 85.80 \\
Absent & \\
American Joint Committee & 11.90 \\
on Cancer clinical stage (\%) & 46.80 \\
IA & 31.00 \\
IB & 10.30 \\
IIA & \\
IIB & \\
\hline
\end{tabular}

analysis (20). Thus, 23 patients in whom SLNs were metastatic by $\mathrm{H} \& \mathrm{E}$ and IHC (conventional histopathology [PATH]) underwent CLND of the affected basin, and 19 of these 23 patients were offered adjuvant therapy with either high-dose (13 patients) or low-dose (6 patients) interferon- $\alpha-2 b$ (IFN, Intron-A; Schering-Plough) (21). However, no lymphadenectomy or adjuvant IFN therapy was offered to patients in whom SLNs were negative by PATH, even in the presence of a positive result at molecular analysis. The followup schedule after primary treatment or staging was reported by Romanini et al. (18). Median follow-up for the entire patient population was $54.8 \mathrm{mo}$ (range, 11.6-80.6 mo).

\section{Lymphatic Mapping and Radioguided SLN Biopsy}

Lymphoscintigraphy was performed $6-18 \mathrm{~h}$ before SLN biopsy as described earlier (18). Briefly, 0.1- to 0.2-mL aliquots containing 4-8 MBq of ${ }^{99 m}$ Tc-albumin nanocolloid (Nanocoll; GE Healthcare) were injected intradermally around the primary melanoma or on skin margins of the surgical scar if the primary lesion had previously been excised (22). Both early dynamic imaging ( 1 frame/min for $30 \mathrm{~min}$ ) and delayed static imaging (various projections, as appropriate) were recorded using a large-field-of-view $\gamma$-camera (Camstar XRT; GE Healthcare) equipped with a parallel-hole, high-resolution collimator $( \pm 10 \%$ window centered on the $140-\mathrm{keV}$ energy peak of ${ }^{99 \mathrm{~m}} \mathrm{Tc}$ ). The skin projection of the SLN was confirmed by external counting with the $\gamma$-probe and marked with indelible ink (22).

SLN biopsy was performed under intraoperative radioguidance (22) using a handheld, thallium-doped cesium iodide-based $\gamma$-probe (Scintiprobe MR 100; Pol.hi.tech). Both the hottest lymph node and any additional radioactive lymph node counting $20 \%$ or more of the 
ex vivo counting rate of the hottest node were harvested for analysis. Removed lymph nodes were labeled as the hottest node or as an additional hot node, as appropriate. When the radiocolloid drained into more than 1 nodal basin, SLN biopsy was performed for each draining basin.

\section{PATH and RT-PCR Analysis}

The harvested SLNs were immediately bisected; one half of each SLN was sent for PATH analysis and the other half was placed in Trizol (Life Technology) for storage at $-80^{\circ} \mathrm{C}$ and delayed RT-PCR molecular analysis.

Details of PATH analysis have been described previously (18). Briefly, 5- $\mu \mathrm{m}$-thick sections were cut from formalin-fixed, paraffinembedded blocks. If $\mathrm{H} \& \mathrm{E}$ staining revealed metastasis, no further staining was performed. If $H \& E$ staining was negative for metastasis, adjacent sections were processed for IHC using antibodies for the S-100 and HMB-45 proteins; further staining with antibody to the MART-1 antigen was performed in case of negative S-100 and HMB-45 staining (23). The entire sections were examined with $40 \times$ and $100 \times$ magnifications to detect discrete foci of tumor cells. The sinusoids were examined at $250 \times$ magnification to detect small clumps or single tumor cells.

For molecular analysis, total RNA was extracted from homogenized tissues and stored in $2-\mu \mathrm{g}$ aliquots under ethanol at $-80^{\circ} \mathrm{C}$ until tested. One microgram of RNA was reverse-transcribed into cDNA in a $20-\mu \mathrm{L}$ reaction mixture containing $100 \mathrm{pmol}$ of random hexamers (Roche), 6 units of reverse transcriptase (Promega), $6.0 \mathrm{mM}$ magnesium chloride, $0.2 \mathrm{mM}$ deoxynucleotide triphosphate, and 8 units of RNase inhibitor (Invitrogen). Samples were sequentially incubated at $25^{\circ} \mathrm{C}(10 \mathrm{~min}), 42^{\circ} \mathrm{C}(60 \mathrm{~min})$, and $95^{\circ} \mathrm{C}(5 \mathrm{~min})$ to inactivate the reverse transcriptase. cDNA samples were subsequently amplified for the target sequence by using the following reaction mixture: $5 \mu \mathrm{L}$ of cDNA, $1.5 \mathrm{mM}$ magnesium chloride, $0.2 \mathrm{mM}$ deoxynucleotide triphosphate, 1 unit of AmpliTaq Gold DNA polymerase (Applied Biosystems), 20 pmol of each primer, and PCR buffer $1 \times$ in $25 \mu \mathrm{L}$ of final volume. Each sample was first tested for amplificability with glyceraldehydes-3-phosphate dehydrogenase primers (yielding a 320-base pair [bp] amplicon (24)) and then analyzed for the melanoma-associated markers Tyr and MART-1 (generating 207- and 364-bp amplicons, respectively $(25,26))$. An ABI 2400 thermal cycler (Thermo Fisher Scientific) was used for amplification, applying the cycling profile described previously (27). After the reaction was complete, $20 \mu \mathrm{L}$ of PCR samples were run on $2 \%$ agarose gel and scored positively when amplicons of the expected size were detected under ultraviolet light.

To minimize the risk of cross-contaminations, RNA extraction, setup of reaction mixtures, and amplifications were performed in separated rooms, and the SLN samples were intermingled with no template samples (negative controls) and RNA extracted from the SK-mel 23 melanoma cell line (positive control).

\section{Classification of SLN by Different Criteria}

Four sets of criteria for classifying SLNs as metastatic or nonmetastatic were derived by variously combining the intraoperative data (ex vivo counting relative to the $20 \%$ threshold) with the results of SLN analysis. The most sensitive indicator available-molecular analysis-was taken as the reference standard for the presence of metastasis in the lymph nodes removed. The following combinations were identified: PATH analysis of only the hottest node, regardless of RT-PCR analysis (scenario A); PATH analysis of all nodes removed, regardless of RT-PCR analysis (scenario B); PATH and PCR analysis of only the hottest node (scenario C); and PATH and PCR analysis of all nodes removed (scenario D).

\section{False-Negative Rate}

The false-negative rate was calculated as the number of falsenegative cases over the sum of the true-positive plus the falsenegative cases (28). True-positive cases were those patients who underwent CLND and in whom the SLN was classified as metastatic by PATH and those patients who did not undergo CLND but were classified as positive by RT-PCR analysis and, during long-term follow-up, presented with locoregional recurrence in a lymphatic basin that had been classified as metastasis-free by PATH analysis. False-negative cases were those patients who, during long-term follow-up, presented with locoregional recurrence in a lymphatic basin that had been classified as metastasis-free by either PATH or RT-PCR analysis. Patients exhibiting distant or in-transit metastasis (without lymph node metastasis in the lymphatic basin of interest) were not considered false-negative cases. False-negative rate was calculated for each of 4 classification scenarios.

\section{Statistical Analysis}

Nonparametric tests were used to compare proportions of reliable SLN biopsies defined according to the 4 different classification scenarios. Data were analyzed using the SPSS/PC+11.5 statistical software (SPSS Inc.), considering a $P$ value of less than 0.05 as significant.

\section{RESULTS}

\section{Classification of SLNs}

A total of 197 SLNs were identified by intraoperative $\gamma$-probe counting ( $84.8 \%$ of which were detected only by preoperative lymphoscintigraphy) in 152 draining nodal basins of the 124 patients ( 1.23 basins per patient, 1.3 SLNs per basin). A total of 131 SLNs in 85 patients did not have metastasis when examined by both PATH and RT-PCR analysis; 24 of 44 harvested SLNs in 23 patients were classified as metastatic by both PATH and RT-PCR analysis; and 17 of 22 harvested SLNs in 16 patients were classified as metastatic by RT-PCR analysis only. No instance in which an RT-PCR-negative SLN was metastatic by PATH analysis was demonstrated, and no significant difference was found in the ex vivo counting rate of negative versus metastatic SLNs (data not shown).

A single SLN was found in 121 of the total 152 draining lymphatic basins, whereas 31 basins had multiple SLNs (a total of 76 SLNs; mean, 2.45 SLNs per basin; range, 2-4 SLNs per basin). Fifteen of 31 lymphatic basins from which more than 1 SLN were harvested harbored metastasis. In 10 of 15 of these tumor-positive basins, metastasis was found in the hottest lymph node, whereas in 5 cases the metastatic lymph node was less radioactive than the hottest node (Fig. 1). Thus, in 5 of 31 basins with multiple SLNs, metastasis was found in a node other than the hottest one (16.1\%). Table 2 shows the location, ex vivo counting rate, and PATH/PCR analysis of these 5 metastatic nodes. Although in 4 of 5 of these nodes metastasis was detected both by PATH and by molecular analysis, it was only RT-PCR that detected metastasis in the remaining node (located in the parotid gland).

Table 3 reports the rates of correct classification for the 41 metastatic SLNs, as derived from the 4 classification scenarios 


\section{Right axilla}

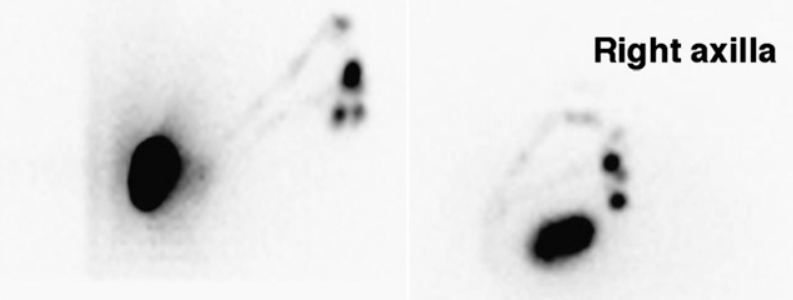

FIGURE 1. Lymphoscintigram (lateral $[\mathrm{A}]$ and oblique anterior [B] projections) obtained from patient who underwent surgery for cutaneous melanoma on her back. Lymph flowed through at least 2 main channels, converging at 4 radioactive nodes in right axilla. After intraoperative $20 \%$ threshold, 4 sentinel nodes were removed surgically. Ex vivo counting rate of third SLN removed (positive for tumor by both PATH and RT-PCR analyses) was equal to $35 \%$ of hottest sentinel node, which, like remaining 2, was negative. This is case in 1 of 5 patients in whom presence of metastasis would have been missed if only hottest node had been harvested for analysis.

(A, B, C, and D). The main determinant of correct classification was molecular analysis, which significantly increased the correct classification rate even if applied to only the hottest node, from $48.8 \%$ of combination A to $87.8 \%$ of combination $\mathrm{C}(P<0.0001)$. On the other hand, when PATH analysis only was considered, removing additional radioactive lymph nodes did not significantly increase the correct classification rate for analyzing only the hottest node (from $48.8 \%$ of combination A to $58.5 \%$ of combination $\mathrm{B}, P=0.5$ ).

\section{Clinical Outcome}

Per current guidelines, CLND was performed only on those 23 patients in whom SLN metastasis was detected by PATH analysis; thus, 16 patients in whom metastasis was detected only by RT-PCR analysis did not undergo CLND of the affected basin. The clinical outcome of the entire patient population followed for a median of $55 \mathrm{mo}$ (range, 12-81 mo) is charted in Figure 2. During long-term follow-up, none of the 23 patients who underwent CLND had locoregional tumor recurrence $(0 \%)$, whereas distant metastasis developed in 15 of these 23 patients and 11 of them died because of such systemic recurrences. By contrast, locoregional recurrence developed in 4 of 16 patients (or 25\%) who had not undergone CLND because metastasis had been detected only by RT-PCR analysis, whereas 2 of these patients had systemic recurrences. Three of 16 patients died in this group ( 2 because of metastatic disease and 1 because of local recurrence that subsequently developed into systemic disease). For the 85 patients in whom SLNs were negative by both PATH and RT-PCR analyses, there was only 1 case of locoregional recurrence (1.2\%), whereas distant metastasis developed in 12 of 85 patients during long-term follow-up, and 9 died.

\section{False-Negative Rate of SLN Biopsy}

A total of 5 locoregional recurrences were observed during follow-up, none of which was in the patients who underwent CLND. All 5 locoregional recurrences were false-negative on PATH analysis, whereas only 1 was false-negative on RT-PCR analysis. The contribution of each set of data to the false-negative rate of SLN biopsy was assessed by modeling different scenarios based on classification of the SLN status according to the 4 classification scenarios (Table 4). The worst scenario was that using the least-sensitive technique of analysis (PATH) to analyze only the hottest lymph node (scenario A). In this case, the false-negative rate of SLN biopsy was 9 of 28 , or $32.1 \%$. Including in the PATH analysis all lymph nodes harvested above the $20 \%$ ex vivo counting rate of the hottest node (scenario B) reduced the falsenegative rate of SLN biopsy to 5 of 28 , or $17.9 \%$. The false-negative rate estimated when using the most-sensitive technique of SLN analysis (RT-PCR) (scenario C), compared with the hottest node only, was 6 of 28 , or $21.4 \%$, whereas the greatest reduction in the false-negative rate was expected when using RT-PCR analysis for all nodes harvested (scenario D) (1 of 28 , or $3.6 \%)$. There was a statistically significant difference only for scenario $\mathrm{D}$ versus scenario A $(P=0.015)$.

\section{DISCUSSION}

The SLN status represents a highly significant prognostic factor in patients with clinically localized cutaneous mela-

TABLE 2

Metastases in Nodes Other Than That with Highest Radioactivity

\begin{tabular}{|c|c|c|c|c|c|}
\hline $\begin{array}{l}\text { Lymphatic } \\
\text { basin }\end{array}$ & $\begin{array}{l}\text { Hottest } \\
\text { node* }^{\star}\end{array}$ & $\begin{array}{c}\text { Metastatic } \\
\text { node }^{\star}\end{array}$ & $\begin{array}{c}\text { Relative to hottest } \\
\text { node }(\%)\end{array}$ & $\begin{array}{c}\text { Order of } \\
\text { harvesting }\end{array}$ & Status \\
\hline Axilla & 1,356 & 1,200 & 88.40 & Second of 3 & PATH+/RT-PCR + \\
\hline Parotid & 68 & 34 & 50.00 & Second of 2 & PATH-/RT-PCR+ \\
\hline Axilla & 832 & 322 & 38.70 & Second of 3 & PATH+/RT-PCR+ \\
\hline Axilla & 455 & 160 & 35.10 & Third of 4 & $\mathrm{PATH}+/ \mathrm{RT}-\mathrm{PCR}+$ \\
\hline Axilla & 900 & 300 & 33.30 & Second of 3 & PATH+/RT-PCR+ \\
\hline
\end{tabular}


TABLE 3

Rates of Correct Classification of Metastatic SLNs

\begin{tabular}{lcc}
\hline Classification & $\begin{array}{c}\text { Number correctly } \\
\text { classified }\end{array}$ & $\begin{array}{c}\text { Correct classification } \\
\text { rate (\%) }\end{array}$ \\
\hline A & $20 / 41$ & 48.80 \\
B & $24 / 41$ & 58.50 \\
C & $36 / 41$ & 87.80 \\
D & $41 / 41^{*}$ & $100.0^{*}$
\end{tabular}

*Standard of reference.

B vs. A, $P=0.5$; C vs. $\mathrm{B}, P=0.005 ; \mathrm{C}$ vs. A, $P<0.0001$; D vs. C, $P=0.06$; D vs. B, $P<0.0001$; D vs. A, $P \ll 0.0001$.

noma $(3,19)$. On average, the SLN is metastatic by conventional PATH analysis in 1 of 5 or 6 patients with stage I-II melanoma. Among the same population with a negative SLN, locoregional recurrence still occurs during follow-up in up to $15 \%$ of cases, which presumably represent failures of nuclear medicine, surgery, or histopathology (8). Although radioguided SLN biopsy has rapidly gained the favor of surgeons, the optimal criteria are still debated both for intraoperative SLN identification and for detection of micrometastasis. Although initial spread of melanoma to regional lymph nodes follows, in general, an orderly pattern, separate parallel draining to more than 1 lymph node (occasionally located in different lymphatic basins) is frequently observed. Routine lymphoscintigraphy is, therefore, important in identifying unsuspected locations of draining lymph nodes, even in patients with melanomas located in the extremities (29).

Radioactive SLNs can readily be identified intraoperatively because of their much higher counting rates relative to background. When histologically positive SLNs are found, they are usually, but not always, the hottest nodes. Several

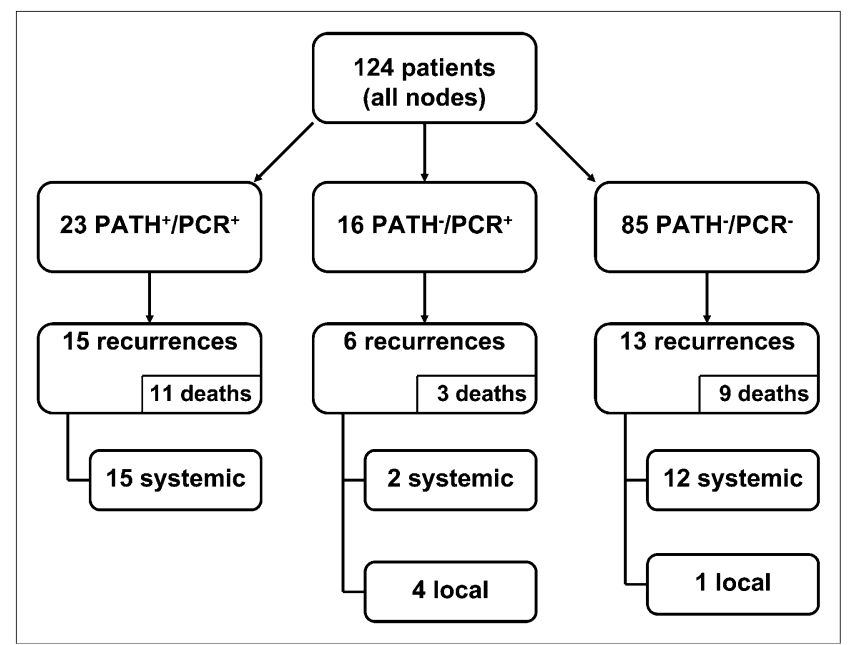

FIGURE 2. Clinical outcome observed in follow-up as function of SLN status evaluated by PATH or by RT-PCR analysis of molecular markers. PATH $-=$ conventional histopathology negative; $\mathrm{PATH}+=$ conventional histopathology positive; PCR - = RT-PCR negative; PCR $+=$ RT-PCR positive.
TABLE 4

Results for the 4 Classification Scenarios

\begin{tabular}{ccccc}
\hline & \multicolumn{4}{c}{ Scenario } \\
\cline { 2 - 5 } Parameter & $\mathrm{A}$ & $\mathrm{B}$ & $\mathrm{C}$ & $\mathrm{D}$ \\
\hline FN cases $(n)$ & $5^{\star}+4^{\dagger}$ & $5^{\star}$ & $2^{\star}+4^{\dagger}$ & $1^{\star}$ \\
TP cases $(n)$ & $19^{\ddagger}$ & $23^{\ddagger}$ & $19^{\ddagger}+3^{\S}$ & $23^{\ddagger}+4^{\S}$ \\
FN rate & $9 /(9+19)$ & $5 /(5+23)$ & $6 /(6+22)$ & $1 /(1+27)$ \\
FN rate $(\%)$ & 32.14 & 17.86 & 21.43 & 3.57
\end{tabular}

*Local recurrences observed.

${ }^{\dagger}$ Additional false-negative cases, which would have been potentially observed if only hottest node had been harvested.

${ }^{\ddagger}$ Patients with SLN classified as metastatic by PATH.

§Patients PATH negative and PCR positive with nodal recurrence during long-term follow-up.

$\mathrm{FN}=$ false-negative; TP = true-positive.

$\mathrm{FN}$ rate $=\mathrm{FN} /(\mathrm{FN}+\mathrm{TP})$.

$\mathrm{FN}$ rate $(\%)=(\mathrm{FN} /[\mathrm{FN}+\mathrm{TP}]) \times 100$.

operational definitions of the SLN have evolved over time to help the surgeon decide exactly which nodes should be removed to maximize the likelihood of locating the true biologic SLN and minimize the superfluous removal of multiple nonSLNs (30-35). An operational SLN definition that was associated with the lowest false-negative rate, using the extensive database of the Sunbelt Melanoma Trial, was described as ". . .all nodes containing radioactive counts $\geq$ $10 \%$ that of the hottest nodes removed" $(7,36)$. However, these authors did not validate such an operational definition with, for example, long-term follow-up for nodal recurrence in basins found to be free from metastasis at SLN biopsy.

Because the $10 \%$ threshold could lead to the superfluous removal of multiple non-SLNs, Coit suggested that such a threshold could be increased to, for example, $20 \%$ or even $30 \%$ (4). Among those thresholds suggested by Coit, for this study we adopted the threshold closest to that proposed by McMasters et al. (i.e., 20\% rather than 10\%) (7) as a new operational definition of SLN. We validated the reliability of this procedure with longterm follow-up for identifying the false-negative cases.

Detection of micrometastatic disease is an additional crucial determinant of the false-negative rate for SLN biopsy in patients with melanoma. In this regard, it is now being increasingly accepted that, despite some controversies still surrounding this issue (37), molecular biology techniques such as RT-PCR support an improvement in sensitivity that seems to come at the cost of increased false-positives and, thus, diminished specificity when compared with histologic analysis. False-positives in tyrosinase mRNA analysis may arise from assay contamination, illegitimate transcription, or legitimate sources such as tyrosinase-expressing Schwann cells and benign nevus cells (15-18). A recent metaanalysis of 22 studies totaling 4,019 patients with clinical stage I or II melanoma who underwent SLN biopsy (38) concluded that detecting SLN metastasis by RT-PCR correlates significantly with the likelihood of disease recurrence. In particular, the 
hazard ratio for disease-free survival and for overall survival is 5-fold and 3-fold, respectively, higher for the patients who were RT-PCR-positive than for those who were RT-PCRnegative. In our study, RT-PCR analysis identified an additional 17 metastatic SLNs over the 24 SLNs that PATH analysis had identified as metastatic.

By combining the ex vivo counting rate with PATH plus RT-PCR analysis, we tested 4 different combinations of SLN status and found that molecular analysis is the most crucial factor for correct classification of SLN status because RT-PCR statistically improves the correct classification rate, even when applied to only the hottest SLN.

The long-term follow-up we adopted (median, $55 \mathrm{mo}$ ) overcomes a potential bias of our study, that is, that we did not harvest for analysis radioactive lymph nodes with counting rates higher than the $10 \%$ threshold proposed by McMasters et al. (7) but lower than the 20\% threshold adopted in this work. In our series, even a $30 \%$ counting rate threshold would have been sufficient for accurately defining the SLN status with PATH analysis, which is currently the recommended operational standard for selecting patients for CLND (Table 4).

Five patients had locoregional recurrences during longterm follow-up; all of these were false-negative cases on PATH, but only 1 was false-negative on RT-PCR analysis. Thus, adding RT-PCR to PATH analysis of all SLNs above the $20 \%$ threshold of the hottest node results in an absolute reduction of the false-negative rate from $17.9 \%$ to $3.6 \%$. On the other hand, if we had removed only the hottest node and used only PATH for analysis (scenario A), we would have potentially observed 4 additional false-negative cases. Added to the 5 local recurrences actually observed, this would have resulted in a $32.1 \%$ false-negative rate, significantly greater $(P=$ 0.015 ) than the value we would expect when analyzing all SLNs with RT-PCR (scenario D). Taken altogether, these observations underscore the importance of not only RT-PCR analysis for a correct classification of the SLN(s) but also the harvesting of all nodes with counting rates above the $20 \%$ threshold relative to the hottest node. Only 1 SLN was detected by lymphoscintigraphy and removed in the single patient who presented locoregional recurrence of the 85 PATH- and PCR-negative cases. This observation suggests a failure of lymphoscintigraphy or RT-PCR analysis or disease in transit.

The results of the Multicenter Selective Lymphadenectomy Trial-I report that SLN status has a significant prognostic power, and lymphadenectomy after detection of clinically occult SLN metastases significantly prolongs disease-free survival, compared with therapeutic lymphadenectomy when SLNs grow to a palpable size (3). Uncertainty still remains as to how to treat PATH-negative and PCR-positive cases. This issue is being addressed by the second Multicenter Selective Lymphadenectomy Trial, designed to evaluate the effect of regional lymphadenectomy on disease-free and overall survival in patients with early nodal metastases detected by RT-PCR analysis (39).

\section{CONCLUSION}

International guidelines lack a unique definition for the surgical detection of SLNs, possibly contributing to the relatively high false-negative rate of SLN biopsy (28). It is, therefore, mandatory for the nuclear medicine community to reach a consensus on the radioactive counting rate threshold to best guide the surgeon in the identification of those lymph nodes with the highest probability of harboring metastases (true biologic SLNs), thus avoiding the unnecessary removal of radioactive non-SLNs and reducing the morbidity related to the procedure. The results of the present study will help the nuclear medicine community to reach such a consensus.

\section{REFERENCES}

1. Lyman GH, Giuliano AE, Somerfield MR, et al. American Society of Clinical Oncology guideline recommendations for sentinel lymph node biopsy in earlystage breast cancer. J Clin Oncol. 2005;23:7703-7720.

2. Gershenwald JE, Uren RF, Mariani G, Thompson JF, Strauss HW. Sentinel lymph node biopsy in malignant cutaneous melanoma. In: Mariani G, Strauss HW, eds. Radioguided Surgery: A Comprehensive Team Approach. New York, NY: Springer; 2008:92-110.

3. Morton DL, Thompson JF, Cochran AJ, et al. Sentinel-node biopsy or nodal observation in melanoma. N Engl J Med. 2006;355:1307-1317.

4. Coit DG. The "true" sentinel lymph node: in search of an operational definition of a biological phenomenon. Ann Surg Oncol. 2001;8:187-189.

5. Morton DL, Bostick PJ. Will the true sentinel node please stand? [editorial]. Ann Surg Oncol. 1999;6:12-14.

6. Nathanson SD. Will the true sentinel node please stand? [letter]. Ann Surg Oncol. 1999;6:514-516.

7. McMasters KM, Reintgen DS, Ross MI, et al. Sentinel lymph node biopsy for melanoma: how many radioactive nodes should be removed? Ann Surg Oncol. 2001;8:192-197.

8. Thompson JF, Stretch JR, Uren RF, Ka VS, Scolyer RA. Sentinel node biopsy for melanoma: where have we been and where are we going? Ann Surg Oncol. 2004;11(suppl 3):147S-151S.

9. Gibbs JF, Huang PP, Zhang PJ, Kraybill WG, Cheney R. Accuracy of pathologic techniques for the diagnosis of metastatic melanoma in sentinel lymph nodes. Ann Surg Oncol. 1999;6:699-704.

10. Cho KH, Hashimoto K, Taniguchi Y, Pietruk T, Zarbo RJ, An T. Immunohistochemical study of melanocytic nevus and malignant melanoma with monoclonal antibodies against S-100 subunits. Cancer. 1990;66:765-771.

11. Walts AE, Said JW, Shintaku IP. Cytodiagnosis of malignant melanoma: immunoperoxidase staining with HMB-45 antibody as an aid to diagnosis. Am J Clin Pathol. 1988;90:77-80.

12. Gadd MA, Cosimi AB, Yu J, et al. Outcome of patients with melanoma and histologically negative sentinel lymph nodes. Arch Surg. 1999;134:381-387.

13. Gershenwald JE, Colome MI, Lee JE, et al. Patterns of recurrence following a negative sentinel lymph node biopsy in 243 patients with stage I or II melanoma. J Clin Oncol. 1998;16:2253-2260.

14. Chao C, Wong SL, Ross MI, et al. Patterns of early recurrence after sentinel lymph node biopsy for melanoma. Am J Surg. 2002;184:520-524.

15. Shivers SC, Wang X, Li W, et al. Molecular staging of malignant melanoma: correlation with clinical outcome. JAMA. 1998;280:1410-1415.

16. Blaheta HJ, Ellwanger U, Schittek B, et al. Examination of regional lymph nodes by sentinel node biopsy and molecular analysis provides new staging facilities in primary cutaneous melanoma. J Invest Dermatol. 2000;114:637-642.

17. Goydos JS, Patel KN, Shih WJ, et al. Patterns of recurrence in patients with melanoma and histologically negative but RT-PCR-positive sentinel lymph nodes. J Am Coll Surg. 2003;196:196-204.

18. Romanini A, Manca G, Pellegrino D, et al. Molecular staging of the sentinel lymph node in melanoma patients: correlation with clinical outcome. Ann Oncol. 2005; 16:1832-1840.

19. Balch CM, Buzaid AC, Soong SJ, et al. Final version of the American Joint Committee on Cancer staging system for cutaneous melanoma. J Clin Oncol. 2001;19:3635-3648.

20. Balch CM, Soong SJ, Gershenwald JE, et al. Prognostic factors analysis of 17,600 melanoma patients: validation of the American Joint Committee on Cancer melanoma staging system. J Clin Oncol. 2001;19:3622-3634. 
21. Kirkwood JM, Strawderman MH, Ernstoff MS, Smith TJ, Borden EC, Blum RH. Interferon alfa-2b adjuvant therapy of high-risk resected cutaneous melanoma: the Eastern Cooperative Oncology Group Trial EST 1684. J Clin Oncol. 1996;14:7-17.

22. Mariani G, Gipponi M, Moresco L, et al. Radioguided sentinel lymph node biopsy in malignant cutaneous melanoma. J Nucl Med. 2002;43:811-827.

23. Prieto VG, Clark SH. Processing of sentinel lymph nodes for detection of metastatic melanoma. Ann Diagn Pathol. 2002;6:257-264.

24. Reinhold U, Ludtke-Handjery HC, Schnautz S, Kreysel HW, Abken H. The analysis of tyrosinase-specific mRNA in blood samples of melanoma patients by RT-PCR is not a useful test for metastatic tumor progression. J Invest Dermatol. 1997;108:166-169.

25. Smith B, Selby P, Southgate J, Pittman K, Bradley C, Blair GE. Detection of melanoma cells in peripheral blood by means of reverse transcriptase and polymerase chain reaction. Lancet. 1991;338:1227-1229.

26. Noppen C, Spagnoli GC, Schaefer C. Isolation of multiple mRNAs from a few eukaryotic cells: a fast method to obtain templates for RT-PCR. Biotechniques. 1996;21:394-396.

27. Pellegrino D, Bellina CR, Manca G, et al. Detection of melanoma cells in peripheral blood and sentinel lymph nodes by RT-PCR analysis: a comparative study with immunohistochemistry. Tumori. 2000;86:336-338.

28. Rossi CR, De Salvo GL, Trifiro G, et al. The impact of lymphoscintigraphy technique on the outcome of sentinel node biopsy in 1,313 patients with cutaneous melanoma: an Italian Multicentric Study (SOLISM-IMI). J Nucl Med. 2006;47:234-241.

29. Uren RF, Howman-Giles RB, Shaw HM, Thompson JF, McCarthy WH. Lymphoscintigraphy in high-risk melanoma of the trunk: predicting draining node groups, defining lymphatic channels and locating the sentinel node. $\mathrm{J} \mathrm{Nucl}$ Med. 1993;34:1435-1440.
30. Morton DL, Wen DR, Wong JH, et al. Technical details of intraoperative lymphatic mapping for early stage melanoma. Arch Surg. 1992;127:392-399.

31. Krag DN, Meijer SJ, Weaver DL, et al. Minimal-access surgery for staging of malignant melanoma. Arch Surg. 1995;130:654-658.

32. Albertini JJ, Cruse CW, Rapaport D, et al. Intraoperative radio-lymphoscintigraphy improves sentinel lymph node identification for patients with melanoma. Ann Surg. 1996;223:217-224.

33. Mudun A, Murray DR, Herda SC, et al. Early stage melanoma: lymphoscintigraphy, reproducibility of sentinel node detection, and effectiveness of the intraoperative gamma probe. Radiology. 1996;199:171-175.

34. Glass LF, Messina JL, Cruse W, et al. The use of intraoperative radiolymphoscintigraphy for sentinel node biopsy in patients with malignant melanoma. Dermatol Surg. 1996;22:715-720.

35. Bostick P, Essner R, Glass E, et al. Comparison of blue dye and probe-assisted intraoperative lymphatic mapping in melanoma to identify sentinel nodes in 100 lymphatic basins. Arch Surg. 1999;134:43-49.

36. Martin RC II, Edwards MJ, Wong SL, et al. Practical guidelines for optimal gamma probe detection of sentinel lymph nodes in breast cancer: results of a multi-institutional study. For the University of Louisville Breast Cancer Study Group. Surgery. 2000;128:139-144.

37. Lugo TG, Braun S, Cote RJ, Pantel K, Rusch V. Detection and measurement of occult disease for the prognosis of solid tumors. J Clin Oncol. 2003;21:2609-2615.

38. Mocellin S, Hoon DS, Pilati P, Rossi CR, Nitti D. Sentinel lymph node molecular ultrastaging in patients with melanoma: a systematic review and meta-analysis of prognosis. J Clin Oncol. 2007;25:1588-1595.

39. Amersi F, Morton DL. The role of sentinel lymph node biopsy in the management of melanoma. Adv Surg. 2007;41:241-256. 